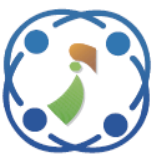

\title{
An Environmental Domain Awareness for Serious-Game Using Perlin Noise Base Heterogeneous Haze Visualization
}

\author{
Fresy Nugroho ${ }^{1,2}$ \\ Eko Mulyanto Yuniarno ${ }^{1}$ \\ Mochamad Hariadi $^{1 *}$ \\ ${ }^{1}$ Department of Electrical Engineering, Institut Teknologi Sepuluh Nopember, Surabaya, Indonesia \\ ${ }^{2}$ Department of Informatics Engineering, Universitas Islam Negeri Maulana Malik Ibrahim Malang \\ * Corresponding author's Email: eman.hanfy@azhar.edu.eg
}

\begin{abstract}
Haze density is critical for heterogeneous haze visualization in developing environmental domain awareness for a serious game. This depiction requires a one-of-a-kind technology that ensures the shifting of heterogeneous haze thicknesses and close to the actual haze. Traditionally the seasoned artists create synthetic haze intuitively. The heterogeneous haze is generated by adding Perlin noise to a homogeneous haze based on the actual haze image density. Perlin noise is used as a heterogeneous haze generator. According to this study's findings, the accuracy is 91.671 percent, the precision is 0.887 , the maximum recall is 0.943 , and the F-measure peak is 0.914 . The proposed method is quick enough to achieve real-time speeds in the range of 10 seconds.
\end{abstract}

Keywords: Environmental domain awareness, Serious-game, Heterogenous haze, Perlin noise.

\section{Introduction}

Environmental domain awareness is the knowledge that something is happening surrounding a person's conditions, affecting a person's growth, health, and progress. Environmental domain awareness, mainly outdoor, can be modeled virtually by surrounding environmental situations like flowing water, rain, flood, and haze. In general, the awareness of the ecological domain, in this case, the haze, has different thicknesses.

In serious games, due to the different thicknesses of the haze, it requires mastery of haze art and takes longer time to create. Every artist or game developer who designs haze has different standards. So it is challenging to approach natural conditions such as those in nature. There is a possibility that even in the same game, the formation of virtual haze has a different degree if it is created by different artist or game developer. Each haze-forming particle has a different density and plays an essential role in producing various haze thicknesses. In the sense that if the haze displayed in a serious game is not close to natural, it will reduce the emotional involvement of players. So that players feel they are not in a serious game environment. Therefore, various methods of generating virtual haze have been developed to increase the emotional involvement of players.

Serious games provide a variety of purposes in addition to amusement, including conveying knowledge and pedagogical values, operating as a training tool, imitating physical world cases [1], inspiring and captivating learners [2]. A serious game, in other words, serves to enhance a player's understanding while also entertaining [3]. Entertainment, on-screen outline, game-play, audiovisual, defiance / complexity, clear / intuitional exhibit, clear intention / target, and game player interest are essential in serious games $[4,5]$.

Researchers look into player modelling [6], and priority-setting challenges affect cognitive functions [7, 8]. Gaming deportment is distinct [9], as evidenced by observable assessments of hostility, demeanor, empathy, and communal skills [10]. The habit of the youngsters may be influenced [11] by the design, judgment [12], and simulated 3D environment game [13]. To increase the quality of serious games, some experts are leveraging literacy, 
climate change engagement [14], the Internet of Things [15], and the realm of disaster risk [16].

The quality of serious games is one of the essential factors impacting scholars' selfadvancement [17]. The screen arrangement influences the scholar's perceptions. The study of serious game and player behaviors is a topic of ongoing research. It is the foundation for developing a powerful serious game programmed for tuition and coaching, because its environment is complex.

An excellent serious game combines three complementary components [18]: the serious component, the harmony item, and the pleasure unit. The education material to practice or teach is the first serious component of the serious game. According to pedagogic principles, this serious component consists of cognitive, emotional, and psychomotor aspects. Depending on pupils' skills, each side has a different percentage.

Second, there is the balancing aspect, which involves matching the game to the pupils' ability. There must be a balance between challenges and skills [19]. Csikszentmihalyi explains the experience of happiness when the job complexity and ability levels are in balance [20]. To avoid boredom, game designers should ensure that the gameplay is varied. During games, exhaustion and boredom can be minimized by shifting tasks and pacing[21, 22]. Furthermore, according to Kwon's finding [23], flow is determined by the game's material, interaction, encouragement, and emotion.

The game is the third half of the enjoyment. While playing, students display much passion. Furthermore, students believe they are not learning [24]. The pleasure requirements are divided into two sections [25], enjoyment and media visualization. The player's involvement and experience, emotional connection [23], and the serious game's flow are all part of the enjoyment side. Meanwhile, in terms of media visualization, enough sound [26] and appealing visual graphic appearance [27-29] are required.

Changes in the gaming environment create attractive visual graphics [30]. The backdrop[30] and native weather situations [31] frequently change the gaming environment. Rain [32, 33], and haze [34] are examples of meteorological conditions that change often. A player's emotions can be dramatized by the dynamic change in a haze [27]. The on-screen outline must be consistent with realistic rendering, which is crucial. As a result, haze simulation based on hazy natural photos is becoming increasingly vital to use.

This research is motivated by the difficulty of driving a car in hazy conditions [35] because driving in hazy lowers the driver's visibility [36]. This article intends to combine the challenge to complete the mission in a hazy condition. They are, furthermore, balancing the game difficulty by adjusting the haze thickness according to the abilities of these students. Environmental domain awareness refers to this shift in the gaming environment. Based on the players' performance, this study provides environmental settings in a thick and thin hazy setting. The haze thickness will increase as the player achieves a high score. The haze will be thinner if the player's score has dropped. Players will notice a reduction in visibility of objects they must acquire as part of a mission due to the thickness change.

The concept of marine domain awareness influenced the concept of Maritime Domain Awareness (MDA). MDA is defined as the use of technology in the maritime environment that affects security, safety, economy, and the environment [37]. By combining intelligence, surveillance, and observation systems, MDA can recognize threats. MDA distinguishes itself by unifying the worldwide marine realm, including ships, cargo, and crew [38]. Obtaining and keeping access to ship data, facilities, infrastructure, and immaterial [39]. A camera or CCTV can be incorporated as a sensor in this experiment. The CCTV can recognize the environmental change. The real environmental change captured by CCTV is used as game environment change. However, the game environment is static and does not respond to changes in the game world. This study suggests an environmental domain awareness design for serious games based on those mentioned earlier.

\subsection{Related work}

In digital scenes, however, modeling and rendering are vital. Simulating the environment, a fictitious three-dimensional stage, or a simulation with fictional components based on real-world events, a real-world scene, or a three-dimensional setting is the most recent trend. Thanks to the Internet, sensors, drones, and other technological breakthroughs, it is now easy for anyone to obtain a photo or video data from numerous realistic circumstances. For reproducing real-world situations, 3D modeling and design are the industry standard. The modeling component is in charge of acquiring the geometrical and lighting features of the scene [40]. Manual modeling with a software geometric modeler, geometry reconstruction from several pictures documenting real-world settings utilizing structurefrom-motion, and texture mapping are examples of traditional modeling methods [38]. The image's rendering component is in charge of creating the 
image from the current perspective, including geometric cut, light measurement, and, in particular, volumetric haze [41].

A volumetric haze method based on a model is more practical and effective. Model-based methods create a hazy visual effect that is consistent, objective, and easy to implement. It is possible to recreate foggy conditions using the haze's physical mechanism model. For the light diffusion model of haze in the atmosphere, [42] started with a single scattering. The hardware is being used in fast render [43]. Mie scattering [44], a typical skylight [45], and heterogeneous hazy pictures based on Perlin noise [46] have all been simulated using real-time simulations. All of the findings are beneficial. The small scale of the environment and the surface features of the objects limit them. These hazy rendering scenes are usually not photo-realistic because they have a high computational cost [47].

\subsection{Contributions and organization of paper}

Visualizing the changing haze is one technique to generate environmental domain awareness in serious games. Perlin noise is applied to homogeneous haze to create heterogeneous haze, allowing for viewing the hazy environment changes. Based on the players' performance, this study provides environmental settings in a thick and thin hazy setting. The haze thickness will increase as the player achieves a high score. The haze will be thinner if the player's score has dropped. Players will notice a reduction in visibility of objects they must acquire as part of a mission due to the thickness change.

The following is a summary of the content of this article. First, it outlines the fundamental ideas and concepts of the physical model of a hazy image, including the Dark channel, Transmission map and density, and Perlin noise. Section 2 outlines the literature review. Section 3 of this article explains the research process. Section 4 discusses the environmental domain awareness design, hazy image data, transmission map, and haze density. Section 5 describes the findings and discussion. Finally, in section 6 , there is a summary of this study.

\section{Preliminaries}

\subsection{Physical model of hazy image}

The color of the original item passes through a hazy medium and scatters, which is then caught by optics, resulting in hazy images. The image's color fades to gray [45]. The scattered light will attenuate and go opposite [48]. When light passes through a scattering medium, it weakens or attenuates along the original route before being distributed in the opposite direction. Eq. (1) shows a mathematical model of the hazy appearance [49].

$$
I(x)=J(x) t(x)+A(1-t(x))
$$

$$
\begin{aligned}
\text { where, } & \\
x & =\text { image with } 2 \mathrm{D} \text { vectors }(\mathrm{x}, \mathrm{y}) \\
I(x) & =\text { Hazy image in RGB } \\
J(x) & =\text { Haze free image in RGB } \\
t(x) & =\text { Transmission Map, from } 0 \text { (total } \\
& \text { haze) to 1 (haze free) } \\
A & =\text { Air light estimation }
\end{aligned}
$$

\subsection{Dark channel}

This section outlines detail about the dark channel. The dark channel describes how one RGB color channel in a few pixels of a picture has a low intensity and even tends to be near to zero. This low intensity falls below a set of criteria:

$$
J_{\text {dark }}(x)=\min _{c \in\{R, G, B\}} J_{c}(x)
$$

If dark pixels in $x$ pixel coordinates are $J_{\text {dark }}$ in Eq. (2), then the dark channel in other pixel coordinates can be expressed as $J_{\text {dark }}(p)$ as long as they match the following requirements:

$$
\min _{c \in\{R, G, B\}} J_{\text {dark }}(p) \leq \min _{c \in\{R, G, B\}} J_{\text {dark }}(x)
$$

If a specific size block window has at least one dark pixel, the criteria in Eq. (3) are satisfied. The block window is defined symbolically as $\Omega$, then:

$$
\min _{p \in \Omega(x)}\left(\min _{c \in\{R, G, B\}} I_{c}(p)\right) \approx 0,
$$

Since the value of $\min _{p \in \Omega(x)}\left(\min _{c \in\{R, G, B\}} I_{c}(p)\right)$ is close to zero, it is abandoned. The symbol $\Omega(x)$ in Eq. (4) represents a window block in the middle of an $x$ coordinate. It means that the bright image as $J$, and the dark channel of image $J$ is:

$$
J_{\text {dark }}(x)=\min _{p \in \Omega(x)}\left(\min _{c \in\{R, G, B\}} I_{c}(p)\right)
$$

The dark channel is the consequence of two minimal operators calculated. The first is the $\min _{c \in R, G, B}$. This operator selects the pixel's most minor RGB color channel. The second operator is $\min _{p \in \Omega_{(x)}}$. The second operator calculates the size of each pixel in the block window. The dark channel 
computation technique for objects in the form of images. The processed image is not required to be bright, which means it does not contain haze. As a result, the dark channel operator is implemented on any image. When the dark channel of a bright picture can be calculated using Eq. (5), the hazy image of $I^{\prime} S$ dark channel can be calculated using Eq. (6):

$$
I_{\text {dark }}(x)=\min _{p \in \Omega(x)}\left(\min _{c \in\{R, G, B\}} I_{c}(p)\right)
$$

Where $I_{\text {dark }}(x)$ represents the image of the haze image's minimal operator computation, $p$ represents the image coordinates at point $p$, and $\Omega_{(x)}$ represents the $x$ coordinates in the block window centered at point $p$. The word " prior " means it was built before the primary process. If the image in the dark channel is:

$$
J_{\text {dark }}(x) \approx 0
$$

The symbol " $\approx$ " in Eq. (7) denotes that the dark channel intensity of each pixel is near zero. This idea was referred to as the dark channel prior. According to research[49], when the sky is used, up to 5,000 dark outdoor imaging channels without a haze reveal that $75 \%$ of dark channel pixels have zero values, while $90 \%$ have intensity levels below 35 . On the other hand, dark haze image channels generate pixels with values high over null. The lowest value of a local block channel is increased by global atmospheric light, which tends to be achromatic with high light intensity, a blend of air-light, and direct attenuation (which tries to provide a gray or white color)[50]. It is concluded that the dark channel's pixel values can be an important factor in estimating haze thickness.

\subsection{Transmission map and density}

Estimation of transmission maps is a problem that could be classified as ill-posed. If the solution to a problem can be more than one and it grows continuously, it is considered an ill-posed problem. Because the inaccuracy of a solution to the problem has such a high value, the concerns are ill-posed [51]. The degree of image transparency [52] or the portion of the not scattered light that reaches the camera [48] is assumed in transmission maps. The transmission map is represented by $t$ with the value [0.1] in the atmospheric scattering model. The transmission map is as light dispersed and passes through the scattering medium until it reaches the observer. As demonstrated in Eq. (8) [34], the estimated transmission map is a mix of depth correlation $\mathrm{d}$ and scattering coefficient:

$$
t(x)=e^{-\beta d(x)}
$$

Adjusting $\beta$ and $\lambda$ producing hazy image simulations with various haze densities. Because in Eq. (9) $\lambda$ is the density/haze density[53], the predicted transmission map has a significant impact on haze density control in the haze simulation scenario. Eq. (9) shows the estimated transmission map[34]:

$$
t(x)^{\lambda}=e^{-(\lambda \beta) d(x)}
$$

\subsection{Perlin noise}

Computer graphics represents natural constructions and textures. One of the most prominent approaches for developing these computer graphic structures [54] is the noise algorithm [50], [51]. Perlin noise is the algorithm used to create native landscapes and clouds. It generates 'characteristics' of variable size (regions of similar value), serving as a versatile pattern and texture building block. Traditional Perlin noise selects vectors from the reduced pile using a hashing algorithm to avoid producing many random vectors. Gradient noise is a technique in which the gradient points are organized pseudo-randomly in space, resulting in interpolation and refinement processes between them. Gradient noise is a fray used in computer graphics that uses a pseudo-random technique.

Each lattice begins with the randomization of the pseudo-random vector gradient and the going vector. At each grid angle, a blue gradient arrow marks the gradient vector. This level is the foundation for random Perlin Noise. The dot product of the gradient vector and the going vector calculated. Eq. (10) shows the heterogeneous haze density distribution [55]:

$$
\operatorname{noise}_{i}(x)=k \times \operatorname{point}(x)
$$

The textural distributions of the haze density are described each by noise $e_{i}(x)$ function. The variable $k$ represents the growth from zero to $i$, while point $(x)$ is an input picture variable that has interpolated.

\section{Environmental domain awareness}

Pixel dimension, image capture length, dark channeling, transmission map, depth map, density map, refined transmission map, and Perlin noise application are all included in the model. This technique calculates and measures the density map to determine the thickness of the haze in the original 


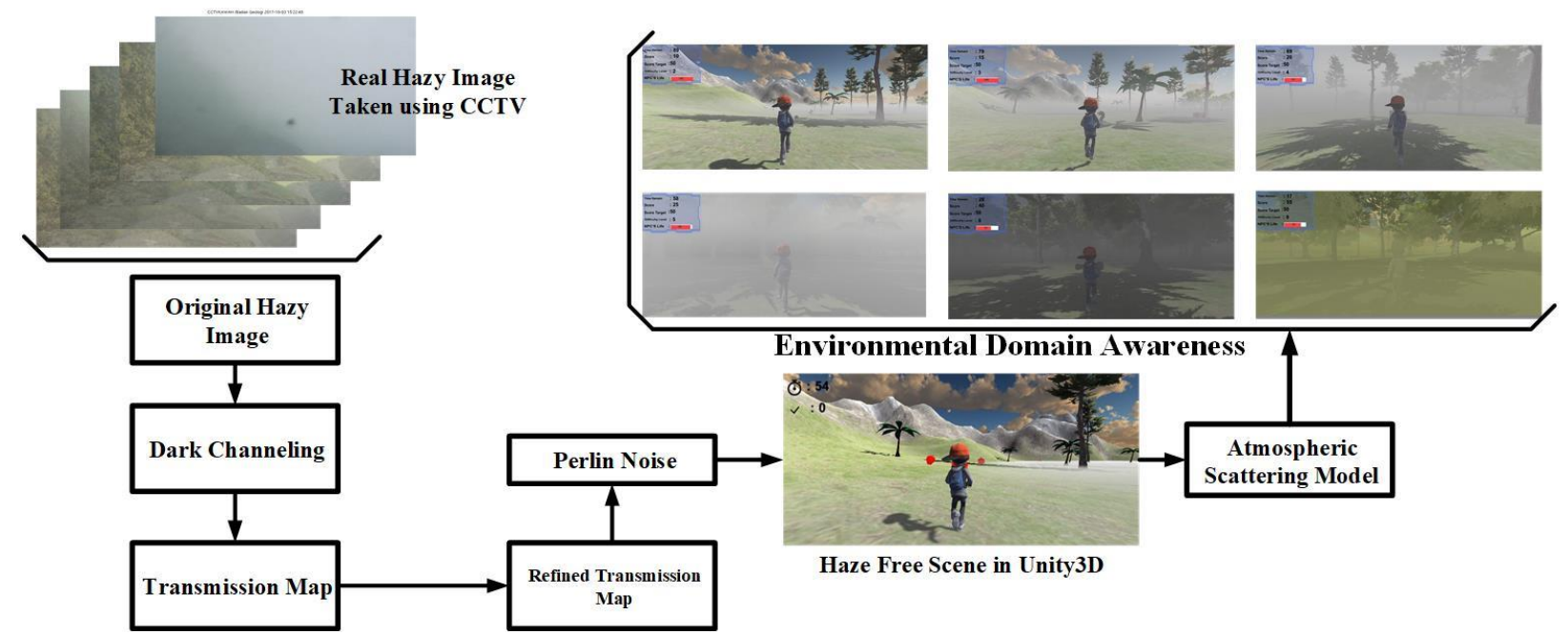

Figure. 1 Proposed environmental domain awareness

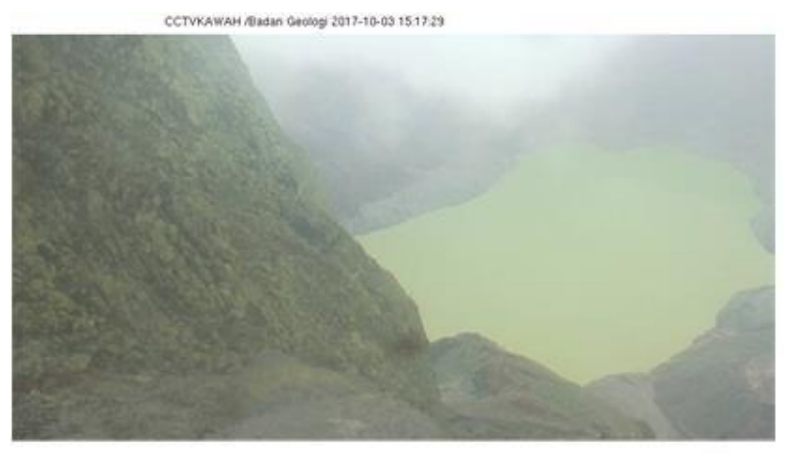

The image on 03 Oct 2017; at 03:17 PM

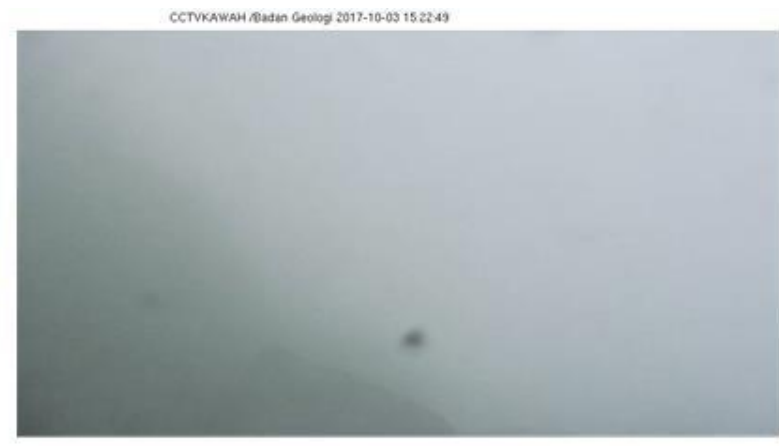

The image on 03 Oct 2017; at 03:22 PM

Figure. 2 Scenes in various condition. Image is taken every 5 minutes

image. The process starts with a dark channeling and transmission map analysis and supporting components like air-light and object depth for a simple, accurate prototype design. Fig. 1 shows the development product.

\subsection{Hazy image data}

The two-dimensional hazy natural outdoor image taken from CCTV at Kelud Mountain [56] was used as a reference. The laboratory conducted the test to measure domain awareness and the haze's opaqueness. Based on the actual hazy image acquired from Kelud mountain, the environmental domain awareness system applies the dark channel, transmission map and calculate haze density. Then, at UNITY 3D, we design a new synthetic haze based on haze density. The test compares the heterogeneous synthetic hazy image to the actual hazy image and the hazy synthetic images created by Sun's, Cao's and Guo.

The CCTV camera Axis Q1755-E Network Camera with a resolution of $1280 \times 720$ pixels, $10 x$ optical magnification, and $12 \mathrm{x}$ digital magnification, total zoom-in 120x, was utilized to collect image data.
From 06.00 AM to 5.00 PM, we acquire hazy imagery in various weather situations. Image sampling occurred every 5 minutes, resulting in 12 photos every hour, for a total of 12 hours per day. For ten days, this investigation used 144 photographs every day. The total number of photographs used in this inspection was 1,440 . This study employs photos with an image size of $800 \times 400$ pixels for all blurry natural and manufactured photographs. Fig. 2 shows a portion of the model. The density $\lambda$ is then

Table 1 . The average hazy image density at every hour

\begin{tabular}{|c|c|}
\hline Time & Average Density $(\lambda)$ \\
\hline $06.00 \mathrm{AM}$ & 3.5537 \\
\hline $07.00 \mathrm{AM}$ & 3.3734 \\
\hline $08.00 \mathrm{AM}$ & 2.4100 \\
\hline $09.00 \mathrm{AM}$ & 3.4243 \\
\hline $10.00 \mathrm{AM}$ & 4.1081 \\
\hline $11.00 \mathrm{AM}$ & 3.2574 \\
\hline $12.00 \mathrm{PM}$ & 2.7610 \\
\hline $01.00 \mathrm{PM}$ & 4.2085 \\
\hline $02.00 \mathrm{PM}$ & 3.0926 \\
\hline $03.00 \mathrm{PM}$ & 8.1813 \\
\hline $04.00 \mathrm{PM}$ & 5.1231 \\
\hline $05.00 \mathrm{PM}$ & 4.1540 \\
\hline
\end{tabular}


Real hazy image

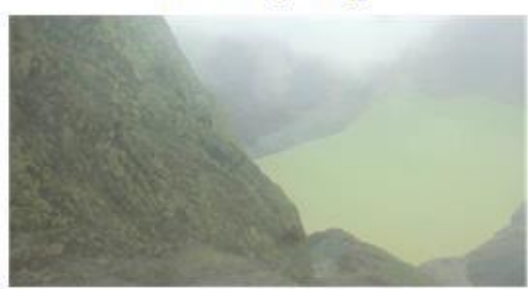

Figure. 3 The depth map and the transmission map for real hazy image at 03 Oct 2017 03:17 PM

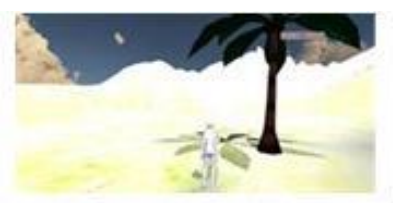

Fog-free-image

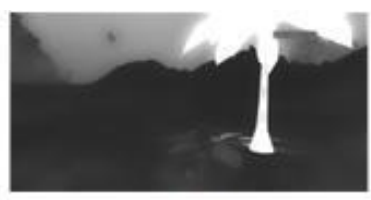

$\lambda=2.41$

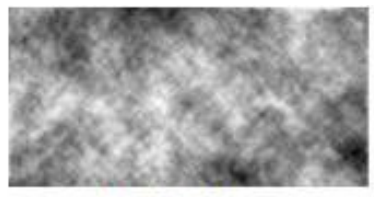

Perlin-Noise
Transmission map
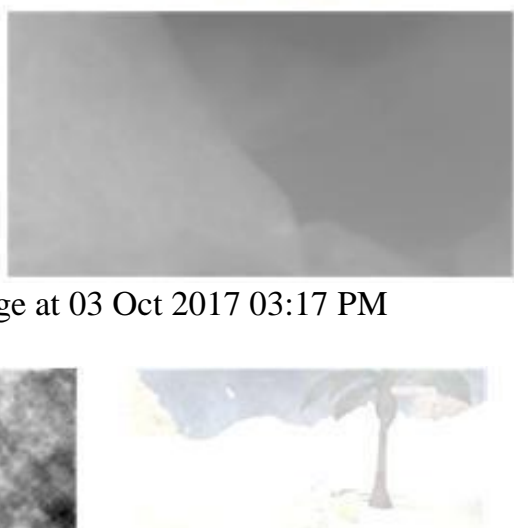

Render-Perlin-Noise

Figure. 4 Render-perlin noise for $\lambda=2.41$, applied to a haze-free image
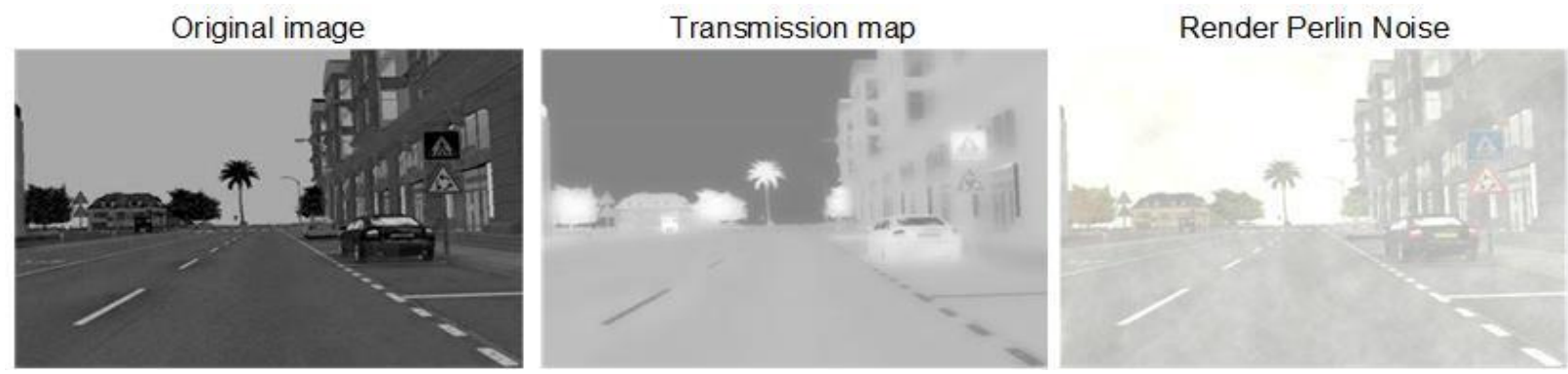

Figure. 5 Render-perlin noise for $\lambda=2.41$, applied to a haze-free image

calculated quantitatively using Haze-Aware Density Estimation [53]. We take an hourly average for ten days of image acquisition. Table 1 shows the average for each hour. At 08.00 AM, the lowest density is 2.41, and at 3.00 PM, the most excellent density is 8.1813 .

\subsection{Depth map and Transmission map}

The first step is to create a depth map from the original hazy image in Fig. 2, based on the value of $d$ in Eq. (8) and an estimated transmission map in Eq. (9).

\subsection{Perlin noise}

In this study, we synthesize Perlin noise for a variety of $\lambda$. As an example, $\lambda=2.41$, shown in Fig. 3.

\section{Result and discussion}

This stage explains how to use the Perlin noise method to create composite synthesis images based on the unequal distribution of haze density. The Sun's, Cao's, and Guo's experiment [34] results compared in this experiment. The confusion matrix is used to contrast synthetic and natural hazes.
The transmission map in Fig. 3 and Perlin noise, implemented to the haze-free image, is used in this study. As a result, we accomplish a render synthetic hazy image for various $\lambda$. The thin haze, for example, represents $\lambda=2.41$, as shown in Fig. 4 .

\subsection{Experimental}

In Fig. 5, the Perlin noise implemented to original haze-free image, based on low density. According to Fig. 5, the haze density distributes unevenly in the heterogeneous synthetic hazy image.

In Fig. 6, we compare Guo's experimental results for the same haze-free image and based on the same lambda. Our experimental results show a more natural hazy image. Guo's road appears to have even thickness, while our results show a natural difference in hazy thickness.

Our experiment showed a more realistic fog image than Sun and Guo's study. The images of roads and distant objects in Sun's research appear dim. In Guo's research, the image of a road and a distant object appears to have the same brightness. Meanwhile, the brightness appears different for roads and distant objects in our research, as shown in Fig. 7. 

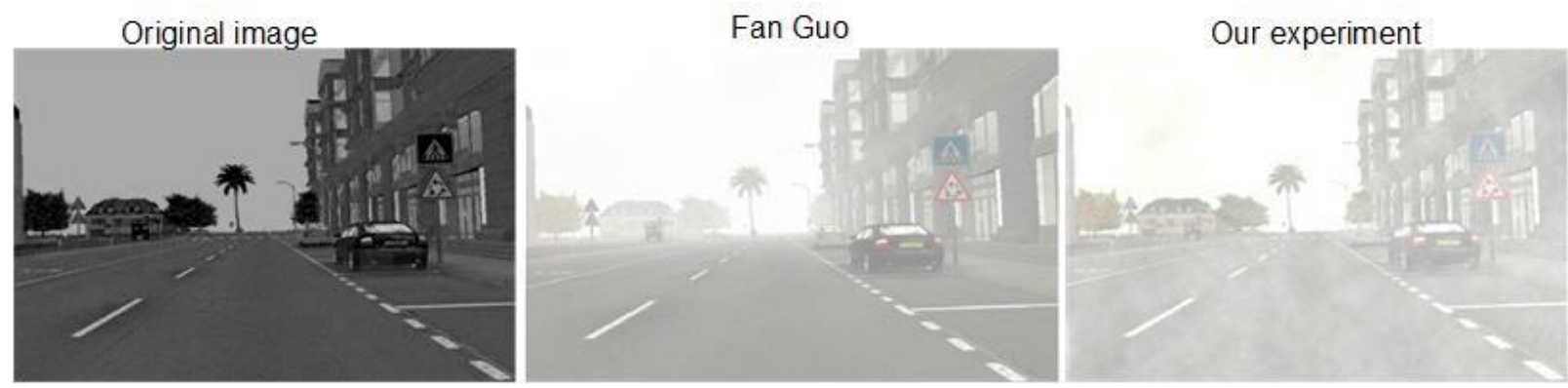

Figure. 6 The comparation between Fan Guo result and our result based on the same $\lambda=2.41$
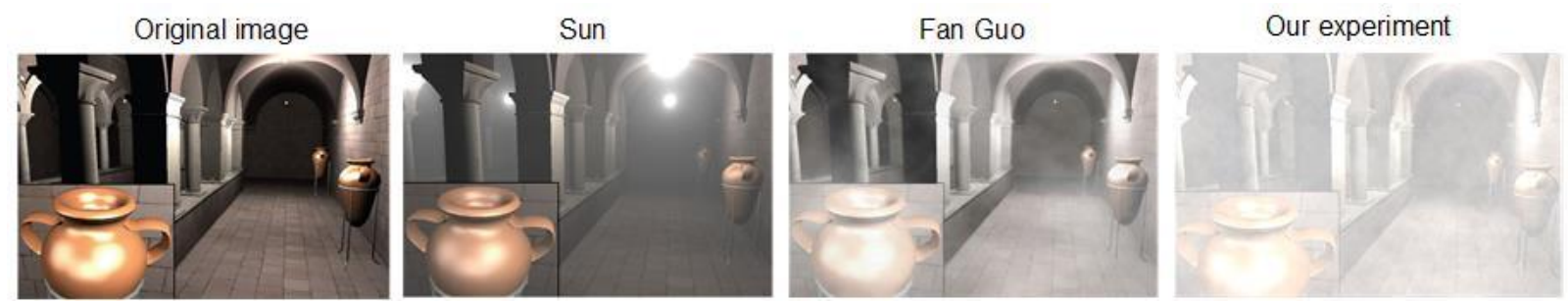

Figure. 7 The comparation between Sun's, Guo's result and our result based on the same $\lambda=2.41$
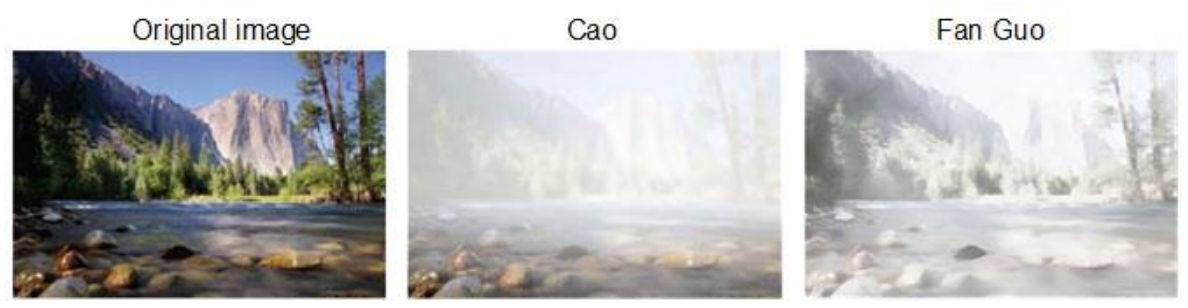

Our experiment

Figure. 8 The comparation between Cao's, Guo's result and our result based on the same $\lambda=2.41$

Compared to Cao and Guo's research for outdoor imagery, our experiment shows a near-realistic haze image. The image of a distant mountain in Sun's research looks bright. Therefore the mountain disappears. In Guo's research, the image of a distant mountain began to disappear, although the edge of the distant mountain started to lose the shadow. While in our research, the image of a distant mountain is still visible, and the details of the shadow on the mountain are still clearly visible. These phenomena are illustrated in Fig. 8.

A confusion matrix reports the comparison results of the heterogeneous hazy synthetic images, and the actual data image consists of 12 pictures for different densities and hours each, displayed in Table 2. This study analyzes the performance of the heterogeneous synthetic hazy image compared to the actual hazy image. Each classification image consists of three haze conditions, such as thin, medium, and thick. Each classification image consists of three conditions of haze, such as thin, medium, and thick.

The diagonal portion shows that the actual positive value for the heterogeneous synthetic hazy image is $91.67 \%$. This value indicates that the heterogeneous synthetic hazy is close to the real haze value. As displayed in Table 3. The performance results in terms of accuracy, precision, recall, and Fmeasure for various synthetic hazy conditions. The accuracy is 91.671 percent, the precision is 0.887 , the maximum recall is 0.943 , and the F-measure peak is 0.914 . It concludes that the heterogeneous synthetic image is close to the hazy natural image.

As a result, Sun's method speed is fast enough to attain real-time rates. Cao's approach takes much longer to compute than Sun's method because the APSF used in Cao's algorithm requires a convolution operation. Therefore its computing speed is linked to convolution mask size and input image size, with relatively high time complexity. Compared to Cao's and Guo's methods, our proposed method is faster, and it takes less time to get a comparable rendered output of the same size in the Matlab. With an image of 800400 pixels, Table 4 displays the computing speeds of the four approaches.

Table 2. The matrix confusion of the heterogeneous synthetic hazy image compare to actual hazy image

\begin{tabular}{|l|c|c|c|c|}
\hline & \multicolumn{4}{|c|}{ Actual hazy image } \\
\hline \multirow{2}{*}{$\begin{array}{l}\text { Heterogeneous } \\
\text { synthetic hazy } \\
\text { image }\end{array}$} & & thin & medium & thick \\
\cline { 2 - 5 } & thin & 4 & 0 & 0 \\
\cline { 2 - 5 } & medium & 0 & 5 & 1 \\
\cline { 2 - 5 } & thick & 0 & 0 & 2 \\
\hline
\end{tabular}


Table 3. The performance of the heterogeneous synthetic hazy image

\begin{tabular}{|c|c|c|c|}
\hline Accuracy & Precision & Recall & F-measure \\
\hline $91.671 \%$ & 0.887 & 0.943 & 0.914 \\
\hline
\end{tabular}

Table 4. shows the rendering times for several rendering methods for $800 \times 400$ pixels

\begin{tabular}{|c|c|c|c|c|}
\hline Method & Sun's & Cao's & Guo's & Our \\
\hline Rendering times & $0.05 \mathrm{~s}$ & $130 \mathrm{~s}$ & $18 \mathrm{~s}$ & $10 \mathrm{~s}$ \\
\hline
\end{tabular}

Table 5. The synthetic haze comparison

\begin{tabular}{|c|c|c|c|}
\hline Reference & Perlin noise & heterogeneous & speed \\
\hline$[34]$ & $\mathrm{x}$ & $\mathrm{x}$ & $18 \mathrm{~s}$ \\
\hline$[45]$ & $\mathrm{x}$ & - & $0.05 \mathrm{~s}$ \\
\hline$[47]$ & $\mathrm{x}$ & - & $130 \mathrm{~s}$ \\
\hline Ours & $\mathrm{x}$ & $\mathrm{x}$ & $10 \mathrm{~s}$ \\
\hline
\end{tabular}

\subsection{Comparison}

Visualizing the shifting haze thickness in a 3D scene is one technique to develop environmental domain awareness in serious games. Perlin noise is used to create heterogeneous haze, which allows for viewing haze environment changes. This research led to the creation of a method for visualizing evolving fog that addresses a variety of heterogeneous haze. Compared to prior synthetic haze implementations in research, this study has some distinct characteristics. These distinctions are stated one by one in Table 5 .

Reference [34] utilized an atmospheric scattering model to construct synthetic homogeneous hazes with varying concentrations on a haze-free nature shot. They use Perlin noise to produce realistic visual effects in computer graphics since natural haze is varied. However, Guo's experimental results are closer to the homogeneous haze image because they are evenly distributed and have better processing time than Cao's experiment. In reference [45], haze transmission map is calculated from the scene's depth, where the attenuation coefficient governs the haze thickness. Lastly, combining illumination and transmission produces a haze effect that results in a blurry image. Sun's experiment reached its highest speed processing time. This speed eliminates image details when covered in a more homogeneous haze. Another group of researchers has proposed a new high realism hazy image for natural scenes, mimicking haze in four different weather scenarios and comparing dehazing approaches. Cao's experiment takes the most prolonged process compared to the other [47].

\section{Conclusion}

The technology that has been developed is useful. It will enable the serious-game industry to build narrative-oriented locations, enhance environmental awareness, minimize operational costs, and increase products' value. The heterogeneous hazy image indicates a 91.671 percent, $0.887,0.943$, and 0.914 accuracies, precision, recall, and F-measure, respectively. Proposed designs can generate a wide range of haze rates with the best haze density and transmission fluctuations. Providing hazy shifting visualization for environmental domain awareness during all serious games should be further considered.

Compared to earlier relevant exploration, the haze changes can be performed accurately and visualiapplied to a serious game $3 \mathrm{D}$ environment.

\section{Conflicts of Interest}

All of the authors certify that they have NO affiliations with or involvement in any organization or entity with any financial interest or non-financial interest in the subject matter or materials discussed in this manuscript.

\section{Author Contributions}

Conceptualization, Fresy Nugroho and Mochamad Hariadi; Data curation, Fresy Nugroho; Formal analysis, Fresy Nugroho and Eko Mulyanto Yuniarno; Investigation, Fresy Nugroho; Methodology, Eko Mulyanto Yuniarno; Software, Fresy Nugroho; Supervision, Eko Mulyanto Yuniarno and Mochamad Hariadi; Writing — original draft, Fresy Nugroho; Writing - review \& editing, Fresy Nugroho, Eko Mulyanto Yuniarno and Mochamad Hariadi.

\section{Acknowledgments}

This research completed in two laboratories, namely in Visicom Engineering Laboratory of Institut Teknologi Sepuluh Nopember Surabaya and Multimedia Laboratory of The Universitas Islam Negeri Maulana Malik Ibrahim Malang.

\section{References}

[1] T. Gunawan, B. Bahari, and M. Kartiwi, "Development of Educational Game for Primary School Mathematics using Microsoft Kinect", Indones. J. Electr. Eng. Comput. Sci., Vol. 6, pp. 457-463, 2017.

[2] S. N. W. Shamsuddin, M. F. Selman, I. Ismail, M. M. Amin, and N. A. Rawi, "A conceptual framework for gamified learning management system for LINUS students", Indones. J. Electr. Eng. Comput. Sci., Vol. 12, No. 3, pp. 13801385, 2018. 
[3] O. B Yedri, E. Lotfi, and M. Bouhorma, "Assessment-driven learning through serious games: Guidance and effective outcomes", Int. J. Electr. Comput. Eng., Vol. 8, pp. 3304-3316, 2018.

[4] A. Belahbib, E. Lotfi, M. Bouhorma, O. B. Yedri, A. Slimani, and E. Fatiha, "Serious Games Adaptation According to the Learner's Performances", Int. J. Electr. Comput. Eng., Vol. 7, pp. 451-459, 2017.

[5] F. Zhao and X. Fang, "Factors Affecting Online Game Players' Loyalty", Internationalization, Design and Global Development, pp. 197-206, 2009.

[6] W. Westera, "Artificial intelligence moving serious gaming: Presenting reusable game AI components", Educ. Inf. Technol., Vol. 25, No. 1, pp. 351-380, 2020.

[7] E. Choi, S. H. Shin, J. K. Ryu, K. I. Jung, S. Y. Kim, and M. H. Park, "Commercial video games and cognitive functions: Video game genres and modulating factors of cognitive enhancement", Behav. Brain Funct., Vol. 16, No. 2, pp. 1-14, 2020.

[8] J. Smith, N. Sears, B. Taylor, and M. Johnson, "Serious games for serious crises: reflections from an infectious disease outbreak matrix game", Global. Health, Vol. 16, No. 1, p. 18, 2020.

[9] N. Männikkö, H. Ruotsalainen, J. Miettunen, H. M. Pontes, and M. Kääriäinen, "Problematic gaming behaviour and health-related outcomes: A systematic review and meta-analysis", $J$. Health Psychol., Vol. 25, No. 1, pp. 67-81, 2020.

[10] S. Kühn, D. T. Kugler, K. Schmalen, M. Weichenberger, C. Witt, and J. Gallinat, "Does playing violent video games cause aggression? A longitudinal intervention study", Mol. Psychiatry, Vol. 24, No. 8, pp. 1220-1234, 2019.

[11] C. Y. Chow, R. R. Riantiningtyas, M. B. Kanstrup, M. Papavasileiou, G. D. Liem, and A. Olsen, "Can games change children's eating behaviour?: A review of gamification and serious games", Food Qual. Prefer., Vol. 80, 2020.

[12] A. H. Aubert, W. Medema, and A. E. J. Wals, "Towards a Framework for Designing and Assessing Game-Based Approaches for Sustainable Water Governance", Water, Vol. 11, No. 4, 2019.

[13] R. Krishnan, S. S, R. Hrishikesh, S. Devadas, G. A, and G. Mannazhath, "A Novel Virtual Reality Game for Disaster Management Applications", pp. 254-257, 2019.
[14] D. Rajanen and M. Rajanen, "Climate change gamification: A literature review", In: Proc. of Gamifin 2019 Conference, pp. 253-264, 2019.

[15] A. K. Adisusilo, M. Hariadi, E. M. Yuniarno, and B. Purwantana, "Optimizing player engagement in an immersive serious game for soil tillage base on Pareto optimal strategies", Heliyon, Vol. 6, No. 3, p. e03613, 2020.

[16] A. S. Nowak, P. Magnuszewski, M. Curl, A. French, A. Keating, J. Mochizuki, W. Liu, R. Mechler, M. Kulakowska, and L. Jarzabek, "An overview of serious games for disaster risk management - Prospects and limitations for informing actions to arrest increasing risk", Int. J. Disaster Risk Reduct., Vol. 31, No. September, pp. 1013-1029, 2018.

[17] A. Abdellatif, B. Mccollum, and P. McMullan, "Serious Games: Quality Characteristics Evaluation Framework and Case Study", In: Proc. of IEEE Integrated STEM Education Conference (ISEC), pp. 112-119, 2018, doi: 10.1109/ISECon.2018.8340460.

[18] P. Caserman, K. Hoffmann, P. Müller, M. Schaub, K. Straßburg, J. Wiemeyer, R. Bruder, and S. Göbel, "Quality Criteria for Serious Games: Serious Part, Game Part, and Balance", JMIR Serious Games, Vol. 8, No. 3, p. e19037, 2020.

[19] M. A. Miljanovic and J. S. Bradbury, "GidgetML: An Adaptive Serious Game for Enhancing First Year Programming Labs", In: Proc. of the ACM/IEEE 42nd International Conference on Software Engineering: Software Engineering Education and Training, pp. 184192, 2020.

[20] M. Csikszentmihalyi, "The Flow, Psychologi \& Happines", The Flow, Psychologi \& Happines, pp. 1-21, 2020.

[21] R. Y. Gomez, J. L. Font, D. C. Caballero, and J. L. Sevillano, "Heuristic usability evaluation on games: a modular approach", Multimed. Tools Appl., Vol. 78, No. 4, pp. 4937-4964, 2019.

[22] A. Thorpe, K. Nesbitt, and A. Eidels, "Assessing Game Interface Workload and Usability: A Cognitive Science Perspective", In: Proc. of the Australasian Computer Science Week Multiconference, pp. 1-8, 2019, doi: 10.1145/3290688.3290749.

[23] C. Kwon, "The Effect of the Degree of Anxiety of Learners during the Use of VR on the Flow and Learning Effect", Appl. Sci., Vol. 10, No. 14, 2020.

[24] F. Nugroho, E. Mulyanto Yuniarno, and M. Hariadi, "Game Based Learning as an Alternative During the Covid-19 Epidemic 
Based on K-13 for Indonesia Elementary School", In: Proc. of International Conference on Engineering, Technology and Social Science (ICONETOS 2020), pp. 210-218, 2021, doi: 10.2991/assehr.k.210421.030.

[25] C. Murphy, D. Chertoff, M. Guerrero, and K. Moffitt, "Design Better Games: Flow, Motivation, and Fun in Learning Games", Design and Development of Training Games: Practical Guidelines from a Multi-Disciplinary Perspective, D. S. Coleman and D. T. Hussain, Eds. Cambridge University Press, pp. 146-178, 2013.

[26] F. Lluís, P. M. Nuevo, M. Møller, and S. Shepstone, "Sound field reconstruction in rooms: Inpainting meets super-resolution", $J$. Acoust. Soc. Am., Vol. 148, pp. 649-659, 2020.

[27] G. Raptis, C. Katsini, C. Fidas, and N. Avouris, "Effects of Image-Based Rendering and Reconstruction on Game Developers Efficiency, Game Performance, and Gaming Experience", In: Proc. of IFIP Conference on HumanComputer Interaction and Lecture Notes in Computer Science, pp. 87-96, 2017.

[28] A. Moncef and A. K. M'hamed, "A Review on 3D Reconstruction Techniques from 2D Images", Innovations in Smart Cities Applications Edition 3, 2020, pp. 510-522.

[29] Z. Kang, J. Yang, Z. Yang, and S. Cheng, "A review of techniques for $3 \mathrm{D}$ reconstruction of indoor environments", ISPRS Int. J. GeoInformation, Vol. 9, No. 5, 2020.

[30] D. Ferdani, B. Fanini, M. Piccioli, F. Carboni, and P. Vigliarolo, "3D reconstruction and validation of historical background for immersive VR applications and games: The case study of the Forum of Augustus in Rome", $J$. Cult. Herit., Vol. 43, 2020, doi: 10.1016/j.culher.2019.12.004.

[31] M. Sajeva, M. Maidell, J. Kotta, and A. Peterson, "An Eco-GAME meta-evaluation of existing methods for the appreciation of ecosystem services", Sustain., Vol. 12, No. 18, 2020.

[32] N. Fan and N. Zhang, "Real-time Simulation of Rain and Snow in Virtual Environment", In: Proc. of the 2012 International Conference on Industrial Control and Electronics Engineering, pp. 29-32, 2012.

[33] P. Goswami, "A survey of modeling, rendering and animation of clouds in computer graphics", Vis. Comput., Vol. 37, pp. 1931-1948, 2021, doi: 10.1007/s00371-020-01953-y.

[34] F. Guo, J. Tang, and X. Xiao, "Foggy scene rendering based on transmission map estimation", Int. J. Comput. Games Technol., Vol. 2014, 2014.

[35] A. Mehra, M. Mandal, P. Narang, and V. Chamola, "ReViewNet: A Fast and Resource Optimized Network for Enabling Safe Autonomous Driving in Hazy Weather Conditions", IEEE Trans. Intell. Transp. Syst., No. August, pp. 1-11, 2020.

[36] F. Rosey, I. Aillerie, S. Espié, and F. Vienne, "Driver behaviour in fog is not only a question of degraded visibility - A simulator study", Saf. Sci., Vol. 95, pp. 50-61, 2017

[37] B. Felsen-parsons, K. Nakamura, S. Fu, and S. Song, "Improving and Operationalizing Maritime Domain Awareness in the South China Sea", 2020. [Online]. Available: https://www.sipa.columbia.edu/file/12428/dow nload?token=LnaLZ2xU.

[38] S. G. Hutchins, S. P. Gallup, D. MacKinnon, G. Schacher, S. Miller, J. Freeman, D. Dunaway, a nd B. Poeltler, "Enhancing Maritime Domain A wareness", 2008. [Online]. Available: http://ww w.dtic.mil/cgi-bin/GetTRDoc?AD=ADA48684 0 .

[39] I. G. B. D. Agastia and A. A. B. Perwita, "Building Maritime Domain Awareness as an Essential Element of the Global Maritime Fulcrum: Challenges and Prospects for Indonesia's Maritime Security", J. Hub. Int., Vol. 6, No. 2, 2017.

[40] N. Snavely, S. M. Seitz, and R. Szeliski, "Photo Tourism: Exploring Photo Collections in 3D", ACM Trans. Graph., Vol. 25, No. 3, pp. 835-846, 2006.

[41] B. Wronski, "Volumetric Fog : Unified compute shader based solution to atmospheric scattering", In: Proc. of The 41st International Conference anf Exhibition on Computer Graphics and Interactive Techniques, pp. 1-67, 2014.

[42] N. L. Max, "Atmospheric Illumination and Shadows", SIGGRAPH Comput. Graph., Vol. 20, No. 4, pp. 117-124, 1986.

[43] Y. Dobashi, T. Yamamoto, and T. Nishita, "Interactive Rendering Method for Displaying Shafts of Light", In: Proc. of Pacific Graphics 2000, pp. 31-435, 2000.

[44] D. Jackèl and B. Walter, "Modeling and Rendering of the Atmosphere Using MieScattering", Comput. Graph. Forum, Vol. 16, No. 4, pp. 201-210, 1997.

[45] R. Ramamoorthi, S. Narasimhan, and S. Nayar, "A Practical Analytic Single Scattering Model for Real Time Rendering", ACM Trans. Graph., Vol. 24, pp. 1040-1049, 2005. 
[46] C. Wang, Z. Wang, and Q. Peng, "Real-time rendering of sky scene considering scattering and refraction", J. Vis. Comput. Animat., Vol. 18, pp. 539-548, 2007.

[47] Y. Cao, W. Shi, and S. Fang, "Simulation of high realism foggy image for natural scene", Vol. 24, pp. 1247-1253, 2012.

[48] R. Fattal, "Single image dehazing", ACM Trans. Graph., Vol. 27, No. 3, 2008.

[49] H. Kaiming, S. Jian, and T. Xiaoou, "Single im age haze removal using dark channel prior", $C v$ pr, Vol. 33, No. 12, pp. 2341-2353, 2009, [Onli ne]. Available: http://kaiminghe.com/publicatio ns/cvpr09.pdf.

[50] S. Lee, S. Yun, J. H. Nam, C. S. Won, and S. W. Jung, "A review on dark channel prior based image dehazing algorithms", EURASIP J. Image Video Process., Vol. 2016, No. 1, pp. 1-23, 2016.

[51] S. I. Kabanikhin and M. A. Shishlenin, "Theory and numerical methods for solving inverse and ill-posed problems", J. Inverse Ill-Posed Probl., Vol. 27, No. 3, pp. 453-456, 2019.

[52] R. T. Tan, "Visibility in bad weather from a single image", In: Proc. of 2008 IEEE Conference on Computer Vision and Pattern Recognition, pp. 1-8, 2008.

[53] L. K. Choi, J. You, and A. C. Bovik, "Referenceless Prediction of Perceptual Fog Density and Perceptual Image Defogging", IEEE Trans. Image Process., Vol. 24, No. 11, pp. 3888-3901, 2015.

[54] X. Min, G. Zhai, K. Gu, Y. Zhu, J. Zhou, G. Guo, $X$. Yang, X. Guan, and W. Zhang, "Quality Evaluation of Image Dehazing Methods Using Synthetic Hazy Images", IEEE Trans. Multimed., Vol. 21, No. 9, pp. 2319-2333, 2019.

[55] K. Perlin, "An Image Synthesizer”, In: Proc. of the 12th Annual Conference on Computer Graphics and Interactive Techniques, pp. 287296, 1985.

[56] N. Fresy, E. M. Yuniarno, M. S. N. Supeno, and M. Hariadi, "Mt. Kelud haze removal using color attenuation prior", IOP Conf. Ser. Mater. Sci. Eng., Vol. 185, p. 12026, 2017. 